\title{
Active Control of Lateral Vibrations of a Railway Carriage
}

\author{
Sven Johansson ${ }^{\dagger}$, Lars Håkansson, Per Persson and Ingvar Claesson \\ Department of Telecommunications and Signal Processing, Blekinge Institute of Technology, \\ S-372 25 Ronneby, Sweden
}

(Received 16 July 2002; accepted 7 October 2002)

\begin{abstract}
As trains are designed for higher and higher speeds the problems of railway carriage vibration are continually on the increase. Lateral vibrations in a railway carriage are noticeable to passengers if the vibration frequencies are lower than approximately $20 \mathrm{~Hz}$. Below this frequency discomfort is a common problem for the passengers and below approximately $1 \mathrm{~Hz}$ motion sickness is a problem. The passive solution of stiffening the carriage chassis to shift the vibrational frequencies higher results in increased manufacturing and running costs, and opposes higher travel speeds due to additional weight. Semi-passive solutions such as modifying the structural dynamics of the carriage body by decoupling heavy underfloor equipment do not reduce the vibrations sufficiently. However, by appending a multi-reference feedforward active vibration control system, one way expect a substantial reduction in the lateral vibration level. Using a dynamic computer model of a railway carriage to simulate the lateral vibration, and using as input bogie acceleration data measured on a running train, multiple-input/singleoutput coherence spectra were shown to constitute a suitable set of reference signals for an active control system. Control simulations based on the Feedforward Multiple-Input/Single-Output Filtered-x LMS Algorithm were carried out using different reference signal combinations. The control results indicate lateral vibration attenuation on the order of $15 \mathrm{~dB}$ at the desired frequency of $10 \mathrm{~Hz}$.
\end{abstract}

${ }^{\dagger}$ Member of the International Institute of Acoustics and Vibration (IIAV)

\section{INTRODUCTION}

As trains are designed for higher and higher speeds the problem of noise and vibration inside railway carriages is continually on the increase. Lateral vibration is noticeable to the passengers if the frequency contents are lower than approximately $20 \mathrm{~Hz}$. Figure 1 shows human perception of lateral vibrations at low frequencies. ${ }^{1}$

The fundamental lateral bending mode is excited by yaw oscillations in the bogies, which occur because of the curved profiles of the contact surfaces of the wheel treads and the rails, i.e. the conicity at the contact points between the wheels and the tracks. The frequency of these bogie oscillations increases with the speed of the train and can be as high as $8 \mathrm{~Hz}$, which approaches the resonant frequency region of the carriage body. Because of the complexity of railway carriage dynamics there is not a single solution to the vibration problem. From a structural point of view, the obvious solution would be to increase the stiffness of the carriage body in order to shift the lowest eigenfrequency to a higher value, thereby decreasing the resonant excitation. This solution has the disadvantage of increasing the weight of the carriage body, thereby adding to the total manufacturing costs. To estimate the cost increase, consider that it takes an extra mass of at least $1000 \mathrm{~kg}$ to increase the lowest eigenfrequency by $1 \mathrm{~Hz}^{2}$ The increased operating costs of a heavier carriage body are more difficult to estimate. However, in view of the intense competition between manufacturers any such costs would be perceived as unfavourable, since such costs are inevitably transferred to the potential customer.

A different approach to minimising the lateral vibrations is to modify the dynamics of the carriage body by decoupling it from heavy underfloor equipment, e.g. the transformer which is normally rigidly mounted under the carriage body. In this approach the transformer becomes a secondary moving mass and can be used to tune the lowest eigenmode of the carriage body. Today this method is used to reduce the vibrations. In order to further reduce the vibration problem while incurring only a minimal increase in the weight of the carriage body, an active control approach can potentially be applied to attenuate the lateral carriage body vibration. The active controller produces opposing forces between the transformer and carriage body, thereby reducing existing vibration. Ideally, a feedforward active control system should be implemented; this necessitates a control reference signal, or a combination of reference signals, which are linearly related to the vibration to be attenuated. ${ }^{3,4}$ The closer the linear relation is between the reference signal and the vibration, the better is the potential performance of the control system.

To choose suitable reference signals, coherence methods can be applied involving the candidate reference signals and the response, i.e. the undesired vibration. Furthermore, the coherence results make it possible to predict the potential effect of feedforward active control of the lateral carriage body vibration before implementation or simulation.

Active vibration control systems are based on either feedforward control or feedback control. ${ }^{3-5}$ Feedforward control relies on the existence of some prior knowledge of the vibration to be controlled; this is contained in a reference signal that drives the secondary source through the controller. Generally speaking, an ideal active controller is of the feedforward type. This type of controller design assumes that the primary source or sources of the undesired vibration can be sensed in order to produce a reference signal, or combination of signals, which is to a high degree correlated with the undesired vibration. -6 $^{4-6}$ 